\title{
Assessment of visual recall and recognition learning in a museum environment
}

\author{
WILLIAM A. BARNARD, ROSS J. LOOMIS, and HENRY A. CROSS \\ Colorado State University, Fort Collins, Colorado 80523
}

\begin{abstract}
Subjects were tested for visual learning of objects in a small museum immediately following either total or limited exposure to the museum environment. Both recall and recognition memory results were better in the limited than in the total exposure condition. Attention time to exhibits correlated positively with recall but showed no relationships with the recognition measure. Findings were discussed in relation to early field research on museum visitor attention patterns.
\end{abstract}

The present study was designed to assess the feasibility of testing for visual recognition and recall memory of visual stimuli in a nonlaboratory setting. A museum provides a good setting for visual memory testing, since a large variety of objects can be used as test stimuli. Museum visitors must process hundreds of items in a manner not considerably different from stimulus presentations in a laboratory experiment. However, one obvious difference is that the visitor, in contrast to the laboratory subject, must locomote through an exhibit environment in order to sequentially experience stimuli. In addition, it is difficult to know for certain what specific stimuli the visitor attends to, since there is not direct control over stimulus presentations. Early researchers in museum visitor behavior unobtrusively observed attention time to different exhibits as an indicator of interest and potential learning (Melton, 1935, Robinson, 1928). It was implicitly assumed that greater attention time would facilitate more learning of objects on display. While contemporary studies of visitor learning have tested for comprehension of semantic material found in label text (Borun, 1977; Screven, 1974), there has not been a follow-up to the original museum attention time studies with direct measures of recall and recognition memory for objects.

Both Melton (1935) and Robinson (1928) felt the patterns of visitor attention were directly related to the educational value of a museum gallery, but they stopped short of suggesting that attention time could be used as an indicator of learning. Direct assessments of learning were not made by these early investigators, but they did raise specific questions about limiting the amount of information available to the visitor. Robinson compared visitor attention times in large and small museums and concluded that the latter provided a more optimal learning opportunity, since attention was given to a higher

The authors are indebted to Brian Moroney and other staff of the Fort Collins Historical Museum for their cooperation in making this study possible. Requests for reprints should be addressed to Ross 3. Loomis, Department of Psychology, Colorado State University, Fort Collins, Colorado 80523. proportion of what was on exhibit and less fatigue would occur. Melton concluded that both the duration of attention to specific objects and the distribution of attention across different exhibits were important variables.

The present study was undertaken to explore whether or not recall and recognition estimates could be made for visual memory of museum stimulus materials. In addition, the study investigated whether limiting information and the amount of attention time paid to exhibits would influence recall and recognition performance.

Recognition and recall memory for specific objects on exhibit were assessed in the study following a visit to a small local history museum. The museum setting used had several hundred objects on display but very few labels installed at the time of the study to minimize semantic cues. As a partial control for attention, subjects were instructed to follow standardized visitation routes. Two levels of information were manipulated by controlling the number of exhibits viewed by subjects.

Previous laboratory research related to learning outcomes using recall and recognition testing procedures has suggested divergent predictions concerning the degree to which subjects would retain visual memory for the objects observed (for review, see Brown, 1976). Three of these were germane to the present study. First, recognition performance with complex pictorial stimuli appears to maintain at high levels even as the number of stimuli is drastically increased (Nickerson, 1968; Shepard, 1967; Standing, Conezio, \& Haber, 1970). Recall performance, however, declines as the amount of information to be learned is increased (Brown, 1976). These typical outcomes suggest that exposure to larger amounts of information in the museum would result in reduced recall performance, although no reduction in recognition would be expected. Second, recognition is reported to remain stable with reductions in exposure time, although recall performance declines readily (Brown, 1976), suggesting that attention time should be positively correlated with recall only. Third, contrary 
to recall performance, higher recognition is reported for novel (low-frequency) than for familiar (highfrequency) stimuli (Gorman, 1961; Kinsbourne \& George, 1974), which should produce a positive correlation of stimulus familiarity with recall and a negative correlation with recognition.

\section{METHOD}

Sixty undergraduate students, in groups of three to four students each, were transported to a local historical museum. Participants were asked to observe all objects in designated exhibits at their own pace and to record the time spent at each exhibit on a schematic diagram of the museum. They were also instructed to provide an estimate of the percent of familiar items in each exhibit case. Familiar items were defined as "items that you have seen before or for which you have seen items that are nearly identical."

The amount of information to be learned was manipulated by providing two exposure conditions. Half of the subjects viewed eight target exhibits in a limited exposure (LE) condition, and the others viewed all 37 exhibits in the museum in a total exposure (TE) condition. Those exhibits not included in the LE condition were shielded from view. All participants were tested with both recall and recognition procedures immediately after looking at the exhibits.

The free recall test required subjects to list as many of the objects in the museum as they could remember, using the schematic diagram as a guide. Results were analyzed in terms of the total percent correctly recalled. The recognition test involved differentiating 57 photographs of objects selected from previously observed exhibits from 57 objects not on exhibit. Exhibited objects photographed were randomly selected from the eight target exhibits viewed by both groups. Distractors photographed were randomly selected from objects kept in storage; they were organized in groupings roughly analogous to the target exhibits. Each item in the recognition test was photographed individually in a separate case with common background and lighting. Results were analyzed in terms of signal detection theory through standard d' scores (Swets, 1964).

\section{RESULTS}

Results supported the expectation that recall performance would be lower in the TE group. Contrary to expectation, recognition was also lower in the $\mathrm{TE}$ condition. These results are summarized in Table 1 . Correlations with attention time revealed a positive relationship with recall for both the $\operatorname{LE}[\mathrm{r}(29)=.45$, $\mathrm{p}<.05]$ and $\operatorname{TE}[\mathrm{r}(29)=.45, \mathrm{p}<.05]$ conditions. Also, recognition performance was not significantly correlated with looking time for $\operatorname{TE}[\mathrm{r}(29)=.26, \mathrm{p}>.05]$ or $\mathrm{LE}$

Table 1

Recognition, Decision Policy, Recall, and Attention Time Results for Total (TE) and Limited (LE) Exposure Conditions

\begin{tabular}{lrrrr}
\hline \multirow{2}{*}{\multicolumn{1}{c}{ Variable }} & \multicolumn{1}{c}{ Mean } & & \\
\cline { 2 - 3 } \multicolumn{1}{c}{ TE } & LE & & df & \multicolumn{1}{c}{$\mathrm{t}$} \\
\hline Recognition $\left(\mathrm{d}^{\prime}\right)$ & .80 & 1.30 & 58 & $3.84 \dagger$ \\
Decision Policy $(\beta)$ & 1.26 & 1.61 & 58 & 1.45 \\
Recall (in Percent) & 13.64 & 27.21 & 58 & $6.90 \dagger \dagger$ \\
Attention Time* & 2.23 & 2.63 & 58 & 1.34 \\
\hline
\end{tabular}

*Estimated average attention time per object (in seconds). $t_{p}<.05$. $\quad t \neq p<.01$. $[\mathrm{r}(29)=.31, \mathrm{p}>.05]$. Correlations of familiarity with both recall and recognition were low and displayed no consistent pattern.

\section{DISCUSSION}

It was possible to obtain recall and recognition estimates for visual memory of museum stimuli. Furthermore, the amount of information attended to by the subjects influenced both measures in a manner consistent with early research. Learning was less for the TE condition. The fact that recognition performance also declined with greater information exposure may point to the role of differences in attention between subjects in laboratory settings and subjects in field settings.

The level of recognition performance shown by subjects was probably influenced by the context in which objects were displayed. In fact, one of the more interesting manipulations studied by Melton (1935) involved changing the groupings of museum objects as a way of altering context. Contemporary investigators have noted the importance of context on recognition memory (e.g., Thomson, 1972). Future work on museum visitor learning will need to consider context as an experimental variable.

Also consistent with earlier work was the finding that duration of attention correlated positively with recall performance. At least in the case of recall measures, it may be possible to consider the attention time observations developed by Melton (1935) and Robinson (1928) as a limited index of visual learning. Results from the present study were insufficient to suggest any conclusions about the role of object familiarity and visual learning.

Because subjects were instructed to use prearranged patterns of exploration, there was a concern that a strong experimenterdemand effect would influence both decision policy and attention time. Inspection of Table 1 reveals that estimated attention time per object was not different for either condition. Also, there was not a significant difference between beta values (Table 1), which would suggest that subjects in the two conditions used similar decision strategies. The magnitude of the beta values reflects a more liberal decision policy, rather than a cautious one. As a further estimate of experimenter demand, the overall attention times to five randomly selected exhibits were compared between subjects in the study and a small sample $(\mathrm{N}=30)$ of visitors observed unobtrusively. Average subject attention times were greater than those of the visitors in four of the five comparisons and significantly different in two of those four comparisons. It would appear that subjects in the study may have demonstrated somewhat longer attention times than do visitors in general.

The research demonstrated that it is possible to make recall and recognition assessments for visual learning of objects in a museum environment. We are currently studying visual learning of museum objects from videotape presentations, which provide for better control over attention. At the same time, there is a need to assess actual visitor learning as a function of the completeness of exploratory patterns used. Previous work has suggested that it is feasible to categorize unobtrusively observed visitor attention patterns on the basis of percentage of museum gallery area attended to by visitors (Melton, 1933).

\section{REFERENCES}

Borun, M. Measuring the immeasurable: A pilot study of museum effectiveness. Washington, D.C: Association of ScienceTechnology Centers, 1977.

Brown, J. Recall and recognition. New York: Wiley, 1976.

Gorman, A. N. Recognition memory for names as a function of abstractness and frequency. Journal of Experimental Psychology, $1961,61,23-24$. 
Kinsbourne, M., \& George, J. The mechanism of the word frequency effect on recognition memory. Journal of Verbal Learning \& Verbal Behavior, 1974, 13, 63-69.

Melton, A. W. Studies of installation at the Pennsylvania Museum of Art. Museum News, 1933, 10, 5-8.

Melton, A. W. Problems of installation in museums of art (Monograph No. 14; New Series). Washington, D.C: American Association of Museums, 1935.

Nicke RSON, R. S. A comment on long term recognition memory for pictorial material. Psychonomic Science, 1968, 11, 58.

RoBINSON, E. S. The behavior of the museum visitor (Monograph No. 5; New Series). Washington, D.C: American Association of Museums, 1928.

Screven, C. G. The measurement and facilitation of learning in the museum environment: An experimental analysis. Washington, D.C: Smithsonian Institution Press, 1974.

ShePARD, R. N. Recognition memory for words, sentences and pictures. Journal of Verbal Learning and Verbal Behavior, $1967,6,156-163$

Standing, L., Conezio, J., \& Haber, R. Perception and memory for pictures: Single trial learning of 2500 visual stimuli. Psychonomic Science, 1970, 19, 73-74.

Swets, J. A. (Ed.). Signal detection and recognition by human observers: Contemporary readings. New York: Wiley, 1964.

Thomson, D. M. Context effects in recognition memory. Journal of Verbal Learning and Verbal Behavior, 1972, 11, 497-511.

(Received for publication August 21, 1980.) 